\title{
ENFERMEDAD PERIODONTAL Y DIABETES: INTERRELACIONES INMUNOLÓGICAS Y CLÍNICAS
}

\section{PERIODONTAL DISEASE AND DIABETES: IMMUNOLOGICAL AND CLINICAL INTERRELATIONSHIPS}

Dra. Alejandra Matejic

\section{INTRODUCCIÓN}

En la cavidad bucal existe una situación única en nuestra biología: la irrupción del órgano dentario, que atraviesa los tejidos conectivo y epitelial creando una solución de continuidad y un serio riesgo para la salud. Como contrapartida a la vulnerabilidad de esta área crítica, nuestro organismo ha desarrollado una serie de mecanismos de adaptación y defensa altamente efectiva. Aún así, en numerosas ocasiones, esta barrera resulta insuficiente y se establece una enfermedad destructiva crónica de los tejidos de soporte dental: la enfermedad periodontal o periodontitis. La etiología primaria de la periodontitis está dada por el biofilm o biopelícula de placa bacteriana, cuya presencia desencadena mecanismos de inmunidad innata que actúan de forma inmediata e inducen una respuesta adaptativa específica. La colonización bacteriana sobre una superficie no descamable, como es el esmalte dental, genera una permanente irritación de los tejidos en el área del surco gingival (Figura 1).

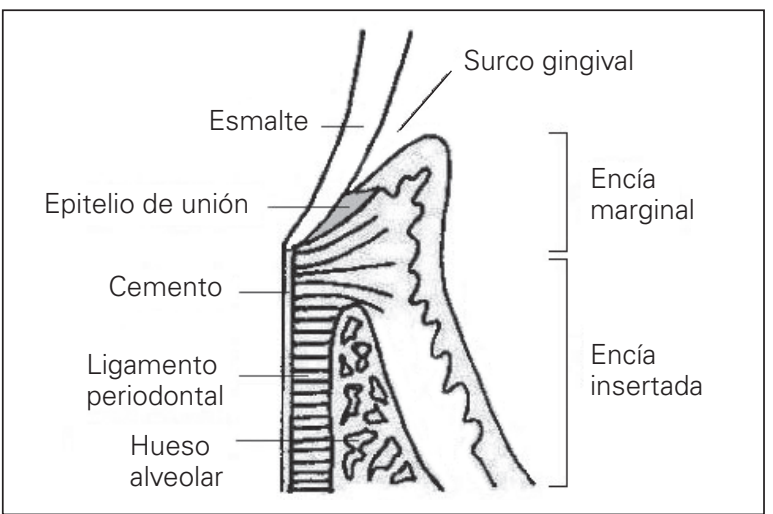

Figura 1: Tejidos periodontales en salud.

Comité de Genética, Inmunología y Prevención. Sociedad Argentina de Diabetes

Magister en Periodontología

Facultad de Odontología. Universidad de Buenos Aires

Correspondencia: Dra. Alejandra Matejic

E-mail: alejandramatejic@gmail.com

Junín $9778^{\circ}$ A (C1113AAC), CABA
Aún en ausencia de inflamación clínica, existe una permanente actividad defensiva, como lo demuestra la presencia de neutrófilos y linfocitos en el tejido conectivo y en el epitelio de unión, así como de un exudado inflamatorio (fluido gingival) que atraviesa estos tejidos y es liberado dentro del surco. La salud periodontal es mantenida entonces gracias a un equilibrio dinámico entre la actividad microbiana y los mecanismos pro y antiinflamatorios del huésped.

\section{Enfermedad periodontal}

La acumulación de placa bacteriana durante un determinado período de tiempo produce modificaciones inflamatorias a nivel gingival (gingivitis), generando una profundización patológica del surco (ahora denominado bolsa) y creando en su interior condiciones de anaerobiosis.

La bolsa gingival se desarrolla como consecuencia del edema y no implica pérdida de inserción, es por ello que el tratamiento de la gingivitis permite la reparación ad integrum de los tejidos, que vuelven a su estado original sin dejar secuelas. Sin embargo si no es tratada, puede persistir como tal en una situación de "aparente equilibrio" o bien, en aquellos pacientes susceptibles, dará lugar al comienzo de destrucción de fibras del ligamento periodontal y reabsorción del hueso alveolar (periodontitis) (Figura 2).

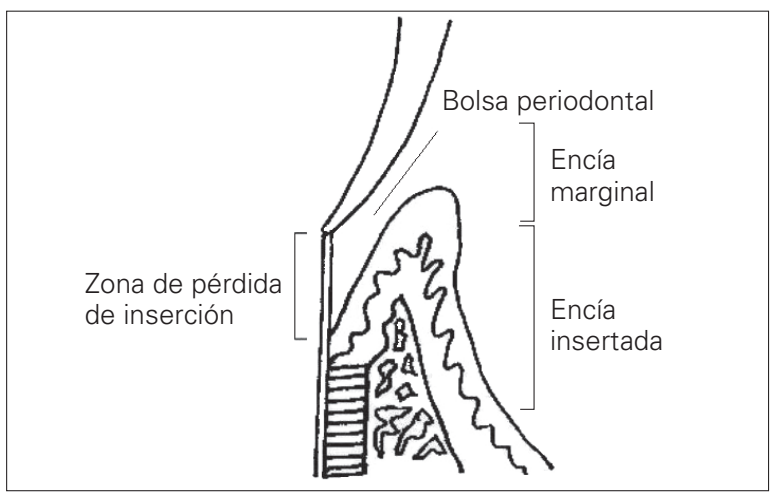

Figura 2: Periodontitis. 
Estudios epidemiológicos coinciden en la alta prevalencia de la gingivitis en poblaciones jóvenes, que oscila entre el 50 y el $100 \%$, mientras que la periodontitis oscilaría entre el 5 y el $50 \%$, encontrándose la mayor prevalencia y severidad en mayores de 45 años ${ }^{1,2}$.

Basado en el estudio epidemiológico NANHES III, realizado entre 1988 y 1994, Albandar concluyó que aproximadamente el $50 \%$ de la población estadounidense mayor de 30 años padecía periodontitis, luego de aplicar un índice de ajuste para corregir el subdiagnóstico ${ }^{3}$.

El inicio de periodontitis, en aquellos pacientes susceptibles, se asocia a un cambio cualitativo del biofilm, que pasa de ser predominantemente Gram positivo y anaerobio facultativo, localizado mayormente a nivel supragingival, a Gram negativo y anaerobio estricto y de mayor desarrollo subgingival. Predominan así bacterias asociadas en forma de complejos, como las Porphyromonas gingivalis, Tannerella forsythia, Prevotella intermedia, Treponema denticola y Fusobacterium nucleatum, entre otras ${ }^{4}$.

Los lipopolisacáridos contenidos en la estructura de la pared celular de los Gram negativos constituyen una potente endotoxina que estimula la respuesta inflamatoria. Sin embargo, la capacidad de estas bacterias para permanecer y generar una infección crónica sugiere que han desarrollado diversos factores de virulencia para evadir los mecanismos de defensa del hospedero, en particular el sistema del complemento y la inmunidad mediada por receptores tipo Toll (TLR), presentes fundamentalmente en células epiteliales, fibroblastos y macrófagos $^{5}$. La activación de receptores tipo Toll (transmembrana), mayormente TLR2 y tipo NOD (citoplasmáticos), resulta en la activación intracelular de factor nuclear kappa-B (NF-kB), que a su vez desencadena la liberación de citoquinas proinflamatorias ${ }^{6}$.

La liberación de citoquinas proinflamatorias, dentro de los tejidos periodontales, es iniciada por numerosas células como los fibroblastos, células endoteliales, macrófagos, osteoclastos, neutrófilos, monocitos, linfocitos y mastocitos. A pesar de que las bacterias organizadas en biofilm constituyen un factor determinante en la etiología de la periodontitis, la activación de los mecanismos inmuno-inflamatorio y sus mediadores, como $\|-1 \alpha\|-,1 \beta, \|-6$, TNF- $\alpha$, prostaglandina E2 (PGE2) y metaloproteinasas de la matriz (MMP), amplifican el proceso destructivo de los tejidos periodontales ${ }^{7}$. De este modo una insuficiente o una exagerada respuesta inmune determinarán la severidad de la enfermedad y la consecuente pérdida de soporte dentario.

\section{Diabetes y periodontitis}

La presencia de diabetes ha sido identificada, por numerosos trabajos de investigación, como un factor de riesgo tanto para la prevalencia como para la severidad y la progresión de la periodontitis ${ }^{8}$ y este riesgo se encuentra en relación directa con la duración de la enfermedad y el control glucémico?.

A su vez, la presencia de enfermedad periodontal no tratada ha demostrado ser un factor que influye negativamente en el control glucémico, mientras que como contrapartida, el tratamiento periodontal mejora el control glucémico y los marcadores sistémicos de inflamación ${ }^{10,11}$.

Los pacientes con periodontitis tienen niveles elevados de citoquinas proinflamatorias en plasma, mientras que los pacientes con diabetes poseen una respuesta inmune exacerbada que potencia estos niveles plasmáticos, favoreciendo la insulinorresistencia. De hecho se ha encontrado un aumento significativo en los niveles de TNF- $\alpha$, II-6 e II-8, en biopsias gingivales alrededor de dientes con periodontitis e implantes con periimplantitis, en pacientes con diabetes y pobre control glucémico ${ }^{12}$.

Según Loos ${ }^{13}$, la bacteriemia generada en las lesiones periodontales es la causa de los cambios en los marcadores sistémicos durante la periodontitis. Y si se calculara el área de superficie de todas las lesiones periodontales en un paciente con periodontitis severa, ésta podría abarcar entre 15 y $20 \mathrm{~cm}^{2}$. Otro investigador calculó un índice que refleja la superficie sangrante en el epitelio de las bolsas periodontales con el fin de cuantificar la dosis de carga inflamatoria. A este índice han logrado relacionarlo significativamente con los valores de $\mathrm{HbA1c}$, independientemente de otros factores ${ }^{14}$.

Se establece así una doble vía entre la EP y la diabetes, ya que al tratarse de dos enfermedades crónicas inflamatorias, existen mecanismos celulares y moleculares que predisponen una a la otra, y su coexistencia genera una asociación cíclica que las potencia ${ }^{15,16,17}$. Numerosos trabajos han establecido asociaciones entre la presencia de enfermedad periodontal y su efecto sobre la salud sistémica ${ }^{18,19,20}$. Este concepto ha dado lugar al desarrollo, en la década de 1990, de una nueva disciplina denominada Medicina Periodontal, incrementando la investigación de diversas patologías sistémicas asociadas, como la diabetes, en- 
fermedades cardiovasculares, enfermedades respiratorias, partos prematuros con bebés de bajo peso, osteoporosis y cáncer, entre otras 21,22,23,24.

La enfermedad periodontal es entonces una infección local de bajo grado con la capacidad de desarrollar una inflamación sistémica de bajo grado que influye en la salud sistémica ${ }^{25}$. La persistencia de una inflamación crónica sistémica puede ocasionar el deterioro de la homeostasis de la glucosa y aumento de la insulinorresistencia, abriendo camino al desarrollo de diabetes tipo $2^{26}$.

\section{Inmunología}

Es conocido el papel que tiene la formación y acumulación de productos finales de la glicosilación avanzada (AGEs), que actúan en forma directa o a través de su interacción con receptores (RAGEs) en el desarrollo de complicaciones vasculares y aumento del stress oxidativo relacionados con la diabetes ${ }^{27,28}$. En realidad, los patrones de reconocimiento involucran a receptores RAGE y TLR en la detección no sólo de patrones moleculares asociados a patógenos (exógenos) sino también de patrones moleculares asociados a daño (proteínas endógenas). La infección se asocia con la liberación de estas proteínas endógenas, como por ejemplo las proteínas del grupo box 1 de alta movilidad (HMGB1). Su unión con los RAGE determina la activación del factor nuclear kappa-B (NF-kB) ${ }^{29,30}$.

Las proteínas HMGB1, también llamadas anfoterinas (ligandos endógenos de RAGE), son liberadas al medio extracelular durante la necrosis o en condiciones de stress, interactuando así además con los receptores TLR. Según los últimos trabajos de investigación, la lista de ligandos intracelulares que interactúan con TLR continúa en aumento ${ }^{31}$.

Los RAGE han sido identificados en múltiples tipos celulares dentro de la encía, incluyendo el endotelio, fibroblastos y células fagocíticas mononucleares, y su expresión se encuentra aumentada en presencia de enfermedad periodontal y más aún en diabetes y otras patologías ${ }^{32}$.

La acumulación de AGEs demostró generar citotoxicidad en los fibroblastos gingivales y mayores niveles de metaloproteinasas de la matriz $(\mathrm{MMP}-1)^{33}$. Estudios in vitro e in vivo sugieren que la interacción AGE-RAGE resulta en marcadas alteraciones en las propiedades de las células endoteliales, células del músculo liso vascular, fagocitos mononucleares y otras células involucradas activamente en la respuesta defensiva frente al biofilm de placa bacteriana.

En modelos animales de diabetes y enfermedad periodontal se comprobó el aumento de acumulación de AGEs y S100 o calgranulina (citoquina proinflamatoria, ligando RAGE) en el tejido gingival, conjuntamente con aumento significativo de los niveles de metaloproteinasas de la matriz (MMP-9). Además, en animales tratados con bloqueantes de RAGE (RAGE soluble) se observó un descenso de hasta el 50-70\% en los niveles de MMP-9, MMP-3 y MMP-2 y niveles de TNF- $\alpha$ similares a los animales con periodontitis sin diabetes ${ }^{34}$.

Un estudio reciente demostró, mediante la utilización de altas dosis de glucosa, la inducción de la expresión de receptores TLR2, en fibroblastos gingivales y la consecuente estimulación de la secreción de NF-kB, TNF- $\alpha$ e IL-1 $\beta$ a través de la proteinquinasa $\alpha$ y $\delta^{35}$.

En los últimos años se comenzaron a estudiar a los inflamasomas, estructuras multiproteicas que actúan como receptores de patrones de reconocimiento intracelular. El inflamasoma NLRP3 es el mejor caracterizado; se activa en presencia de señales de stress celular, virus, bacterias y sus productos, y juega un rol central en la enfermedad periodontal como lo demuestran trabajos in vitro realizados en cultivos de fibroblastos, linfocitos y monocitos/ macrófagos, con diferentes biofilms subgingivales ${ }^{36,37,38,39}$. El inflamasoma NLRP3, además, ha sido implicado en la secreción de II-1 $\beta$ bioactiva, apareciendo como un sensor importante en la disregulación metabólica, el control de la insulinorresistencia asociada a obesidad y la disfunción de las células $\beta$ pancreáticas ${ }^{40}$. Por último, las alteraciones en el metabolismo de los lípidos, relacionadas con diabetes, se han correlacionado con una alteración en la función de los monocitos/macrófagos tanto in vitro como in vivo, dando lugar a una mayor producción de citoquinas proinflamatorias. También se ha postulado que esta activación de los monocitos podría generarse por el stress oxidativo inducido por la interacción AGE-RAGE, provocando mayor secreción de TNF- $\alpha, \|-1 \beta$ e II- 6 , relacionadas con inflamación y destrucción de los tejidos periodontales.

Si bien la periodontitis induce stress oxidativo localmente en los tejidos periodontales, un trabajo de Allen y colaboradores comprobó un aumento en plasma de biomarcadores y de stress oxidativo en pacientes con diabetes tipo 2 y enfermedad periodontal, sugiriendo que esta última amplificaría la disfunción de las células $\beta$. Además, en di- 
chos pacientes, se encontraron los menores valores de HDL y los mayores de triglicéridos, aunque con diferencias no significativas ${ }^{41}$.

En algunos casos, se ha reportado la disminución en la función de quimiotaxis y fagocitosis en los leucocitos polimorfonucleares neutrófilos que haría menos eficaz la respuesta protectiva frente al biofilm de placa bacteriana ${ }^{42,43}$. Contrariamente se ha observado un incremento de proteína quimiotáctica de monocitos (MCP-1) en tejidos periodontales, tanto en diabetes como en periodontitis, en animales de laboratorio ${ }^{44}$. En pacientes con periodontitis se ha observado un aumento significativo de MCP-1 y su disminución postratamiento, tanto en fluido gingival como en saliva y plasma ${ }^{45}$.

\section{Clínica}

Desde el punto de vista clínico, no existen diferencias entre la periodontitis en pacientes diabéticos y pacientes sistémicamente sanos. Sin embargo, el control metabólico ha demostrado ser determinante en la severidad de la destrucción periodontal y en la respuesta al tratamiento ${ }^{46}$.

Se ha reportado la formación de abscesos periodontales crónicos múltiples y recurrentes, rápida destrucción de hueso alveolar o cicatrización demorada luego del tratamiento en pacientes diabéticos con pobre control metabólico ${ }^{47}$, razón por la cual el periodoncista podría sospechar de la presencia de diabetes no diagnosticada o mal controlada, diferenciando aquellos pacientes diagnosticados y con buen control glucémico ${ }^{48}$.

El tratamiento de la periodontitis consiste básicamente en el raspaje y alisado de las raíces dentarias en los sitios afectados, logrando así la eliminación del biofilm subgingival junto con los depósitos de cálculo y la eliminación del cemento radicular infectado, dejando una superficie lisa apta para la reparación de los tejidos. Numerosos estudios han implementado la administración de antibióticos sistémicos, en conjunto o con posterioridad a la terapia mecánica, logrando mejores resultados clínicos ${ }^{49}$. Su uso está recomendado en periodontitis crónicas severas y periodontitis agresivas. En estos casos, los antibióticos de elección son amoxicilina y metronidazol utilizados en combinación ${ }^{50,51}$.

Los pacientes periodontales con diabetes bien controlada, en la mayoría de los casos, pueden recibir el mismo tratamiento que aquellos sistémicamente sanos.

Las tetraciclinas y sus análogos, utilizados en ratas, han demostrado capacidad de inhibir a las colagenasas, las metaloproteinasas de la matriz y la reabsorción ósea ${ }^{52}$. La doxiciclina es un antibiótico que ha sido utilizado en bajas dosis, en casos de enfermedad periodontal y diabetes, con el fin de aprovechar su doble acción como antimicrobiano y modificador de la respuesta del huésped ${ }^{53}$. En un estudio clínico utilizando doxiciclina 100 mg/ día durante 2 semanas, a partir de la terapia básica periodontal, se logró una reducción significativa en los valores de $\mathrm{HbA} 1 \mathrm{c}$ a los 3 meses, mientras que el grupo control (sin antibiótico) mantuvo estos valores sin cambios ${ }^{54}$. Otro estudio donde se realizó tratamiento periodontal aunque sin la utilización de antibióticos, resultó sin mejora en los valores de $\mathrm{HbA} 1 \mathrm{c}$, mientras que llevó a una reducción en los niveles de proteína $\mathrm{C}$ reactiva ${ }^{55}$.

Tervonen y colaboradores no encontraron diferencias en el resultado de tratamiento periodontal no quirúrgico entre pacientes diabéticos tipo 2 y no diabéticos ${ }^{56}$.

Si bien al comparar estudios clínicos los resultados son variables y a veces contradictorios, esto podría deberse a la falta de estandarización en cuanto a tipos de diabetes, tiempo de evolución de la enfermedad, controles metabólicos y presencia de complicaciones relacionadas. De todos modos la evidencia indica que el buen control metabólico es el parámetro más indicado para esperar no sólo una buena respuesta al tratamiento periodontal sino además que el porcentaje de recurrencia de periodontitis en pacientes tratados sea similar en individuos con y sin diabetes ${ }^{57}$. Estudiando pacientes con diabetes tipo 1 con al menos 10 años de evolución, se observó que la severidad de la periodontitis aumentaba conforme con la severidad de complicaciones en diferentes órganos, encontrándose mayor sangrado al sondaje, mayor profundidad de sondaje y mayor pérdida de inserción que en aquellos pacientes sin complicaciones o con complicaciones incipientes. La existencia de complicaciones avanzadas fue además el único factor relacionado a la diabetes que resultó predictor de bolsas periodontales mayores o iguales a $4 \mathrm{~mm}$. La retinopatía resultó ser la complicación más adecuada para comparar porque suele aparecer en primer lugar y puede clasificarse desde no existente hasta severa ${ }^{58}$.

Lei Chen y colaboradores estudiaron los diferentes índices periodontales en pacientes con diabetes tipo 2 y encontraron una correlación positiva 
significativa entre la profundidad de sondaje y los valores de $\mathrm{HbA} 1 \mathrm{c}$ y niveles de proteína $\mathrm{C}$ reactiva, mientras que no hallaron correlación con los niveles séricos de TNF- $\alpha$, glucemia en ayunas ni perfiles lipídicos ${ }^{59}$. Si bien los niveles de TNF- $\alpha$ no pudieron correlacionarse con la severidad de la enfermedad periodontal, los valores circulantes resultaron más altos debido a su triple origen: el tejido adiposo, la estimulación de monocitos-macrófagos a través de los AGEs y la inflamación periodontal.

Asimismo, en un estudio prospectivo realizado en la ciudad de Hisayama, Japón, se relacionó el estado periodontal y la tolerancia oral a la glucosa en 961 adultos, encontrando que la mayor profundidad al sondaje fue también el parámetro que estaba significativamente asociado con el desarrollo de intolerancia y diabetes ${ }^{60}$. A lo largo de 10 años, 415 personas con tolerancia normal a la glucosa desarrollaron intolerancia o diabetes. Aquellos con bolsas más profundas tuvieron un aumento significativo en los valores de HbA1c y cada milímetro de profundidad adicional se correspondió con un aumento del $0,13 \%$ de HbA1c. De modo que la inflamación crónica generada por patógenos periodontales en bolsas profundas afectaría el control glucémico en individuos no diabéticos. Una hipótesis sería que la producción de TNF- $\alpha$ originada por el estímulo continuo de los lipopolisacáridos de bacterias gram negativas, aumentaría la insulinorresistencia. Se sugiere entonces una influencia directa del estado periodontal en la etiología de la diabetes tipo 2 y sus estados previos.

En síntesis, la enfermedad periodontal genera una inflamación de bajo grado que aumenta la producción de citoquinas que favorecen la insulinorresistencia, aumentando el riesgo de desarrollar diabetes tipo $2^{61}$.

\section{DISCUSIÓN}

La enfermedad periodontal y la diabetes son enfermedades inflamatorias crónicas, y como tales comparten numerosos mecanismos biológicos. Existen evidencias que sugieren que la presencia de una de ellas puede favorecer el desarrollo de la otra, y que su coexistencia produce la potenciación de sus efectos, generando mutuamente una influencia negativa en su evolución. Desde el punto de vista clínico, comparten además la característica de ser asintomáticas y por ello, tanto la diabetes tipo 2 como la enfermedad periodontal suelen cursar encubiertas durante varios años an- tes de ser diagnosticadas. Los controles médicos y odontológicos resultan imprescindibles para lograr el diagnóstico precoz e implementar el tratamiento adecuado con el fin de mejorar las expectativas de evolución de estas patologías.

\section{REFERENCIAS}

1. Brown LJ., Oliver RC., Loe H. Periodontal disease in the US in 1981: prevalence, severity, extent and role in tooth mortality. J Periodontol. 1989; 60:363-370.

2. Brown LJ., Loe H. Prevalence, extent, severity and progression of periodontal disease. Periodontology 2000, 1993; 2:57-71.

3. Albandar JM. Underestimation of periodontitis in NHANES surveys. J Periodontol. 2011 Mar; 82 (3):337-341.

4. Socransky SS, Haffajee AD, Cugini MA, Smith C, Kent RL Jr. Microbial complexes in subgingival plaque. J Clin Periodontol. 1998; 25:134-144.

5. Krauss JL, Potempa J, Lambris JD, Hajishengallis G. Complementary Tolls in the periodontium: how periodontal bacteria modify complement an TLR responses to prevail in the host. Periodontology 2000. Vol. 52; 2010, 141-162.

6. Zelkha SA, Freilich RW, Amar S. Periodontal innate inmune mechanisms relevant to aterosclerosis and obesity. Periodontology 2000, Vol. 54, 2010: 207-221

7. Preshaw PM, Taylor JJ. How has research into cytokine interactions and their role in driving immune responses impacted our understanding of periodontitis? J Clin Periodontol. 2011; 38 Suppl 11:60-84.

8. Papapanou PN. World workshop in clinical periodontics. Periodontal diseases: Epidemiology. Ann Periodontol 1996; 1:1-36.

9. Tervonen T, Knuuttila M. Relation of diabetes control to periodontal pocketing and alveolar bone level. Oral Surg Oral Med Oral Pathol. 1986; 61:346-349.

10. Stewart JE, Wager KA, Friedlander $\mathrm{AH}$, Zadeh $\mathrm{HH}$. The effect of periodontal treatment on glycemic control in patients with type 2 diabetes mellitus. J Clin Periodontol. 2001; 28:306-310.

11. Grossi SG, Skrepcinski FB, DeCaro T, Robertson DC, Ho AW, Dunford RG, et al.Treatment of periodontal disease in diabetics reduces glycated hemoglobin. J Periodontol. 1997; 68:713-719.

12. Venza I, Visalli M, Cucinotta M, De Grazia G, Teti D, Venza M. Proinflamatory gene expression at chronic periodontitis and peri-implantitis sites in patients with or without type 2 diabetes. J Periodontol. Jan 2010; 81 (1):99-108.

13. Loos BG. Systemic markers of inflammation in periodontitis. $J$ Periodontol. 2005; 76 (11 Suppl):2106-2115.

14. Nesse W, Linde A, Abbas F, Spijkervet FK, Dijkstra PU, de Branbander EC, Gerstenbluth I, Vissink A. Dose-response relationship between periodontal inflamed surface area and $\mathrm{HbA} 1 \mathrm{c}$ in type 2 diabetics. J Clin Periodontol. 2009; 36:295-300.

15. Mealey BL. Periodontal disease and diabetes. A two-way street. J Am Dent Assoc. 2006 Oct; 137 Suppl:26-31.

16. Nishimura F, Iwamoto $Y$, Mineshiba J, Shimizu A, Soga Y, Murayama $Y$. Periodontal disease and diabetes mellitus: the role of tumor necrosis factor-alpha in a 2-way relationship. J Periodontol. 2003; 74:97-102.

17. Kim J, Amar S. Periodontal disease and systemic conditions: a bidirectional relationship. Odontology. 2006; 94:10-21.

18. Slots J. Casual or causal relationship between periodontal infection and non-oral disease? J Dent Res. 77 (10) 1998; 1764-1766.

19. Scannapieco FA. Position Paper. Periodontal disease as a potential risk factor for systemic diseases. J Periodontol. 1998; 69 (7): 841-850. 
20. Williams RC, Barnett AH, Claffey N, Davis M, Gadsby R, Kellett M, Lip GY, Thackray S. The potential impact of periodontal disease on general health: a consensus view. Curr Med Res Opin 2008; 24:1635-1643.

21. Rose LF, Genco RJ, Cohen W, Mealey BL. Periodontal Medicine, 2000. B.C. Decker Inc.

22. Williams RC, Offenbacher S. Periodontal medicine: a new branch of periodontology. Periodontology 2000. 23; 2000, 9-12.

23. Williams RC. Periodontal disease: the emergence of a new paradigm. Compend Contin Educ Dent. Special Issue. 2001; 22 (2); 3-6.

24. Slots J, Kamma J. General health risk of periodontal disease. Int Den J 2001; 51:417-427.

25. Abhijit N Gurav. Periodontitis and Insulin Resistance: Casual or Causal Relationship? Diabetes Metab. J 2012; 36:404-411.

26. Shoelson SE, Lee J, Goldfine AB. Inflammation and insulin resistance. J Clin Invest. 2006; 116:1793-1801.

27. Vlassara H. Advanced glycation end-products and atherosclerosis. Ann Med. 1996 Oct; 28 (5): 419-426.

28. Lalla E., Lamster IB., Drury S., Fu C., Schmidt AM. Hyperglycemia, glycoxidation and receptor for advanced glycation endproducts: potential mechanisms underlying diabetic complications, including diabetes-associated periodontitis. Periodontology 2000, 2000, 23; 50-62.

29. Van Zoelen MA, Achouiti A, van der Poll T. RAGE during infectious diseases. Front Biosci (Schol Ed), 2011 Jun; 1 (3):1119-1132.

30. Nogueira-Machado JA, Volpe CM, Veloso CA, Chaves MM. HMGB1,TLR and RAGE: a functional tripod that leads to diabetic inflamation. Expert OpinTherTargets 2011 Aug; 15 (8):1023-1035.

31. Lin L, Park S, Lakatta EG. RAGE signaling in inflammation and arterial aging. Front Biosci 2009 Jan; 1 (14):1403-1413.

32. Katz J, Wallet S, Cha S. Periodontal disease and the oral-systemic connection: "Is it all the RAGE?". Quintessence Int. 2010, 41; 3:229-237.

33. Yu S, Li H, MaY, Fu Y. Matrix metalloproteinase-1 of gingival fibroblasts influenced by AGEs and their association with receptor for AGEs and nuclear factor-kB in gingival connective tissue. J Periodontol. 2012, 83; 1: 119-126.

34. Lalla E, Lamster IB, Stern DM, Schmidt AM. Receptor for advanced glycation end products, inflammation and accelerated periodontal disease in diabetes: mechanisms and insights into therapeutic modalities. Ann Periodontol 2001; 6:113-118.

35. Jiang SY, Wei CC, Shang TT, Lian Q, Wu CX, Deng JY. High glucose induces inflammatory cytokine through protein kinase $\mathrm{C}$-induced toll-like receptor 2 pathway in gingival fibroblasts. Biochem Biophys Res Commun. 2012 Oct 26; 427 (3):666-670.

36. Jun HK, Lee SH, Lee HR, Choi BK. Integrin a5b1 activates the NLRP3 inflammasome by direct interaction with a bacterial surface protein. Immunity, 2012 May 25; 36 (5):755-768.

37. Georgios N. Belibasakis, Anders Johansson. Aggregatibacter actinomycetemcomitans targets NLRP3 and NLRP6 inflammasome expression in human mononuclear leukocytes. Cytokine, 2012; 59:124-130.

38. Bostanci N, Meier A, Guggenheim B, Belibasakis GN. Regulation of NLRP3 and AIM2 inflammasome gene expression levels in gingival fibroblasts by oral biofilms. Celular Immunology, 2011; 270: 88-93.

39. Belibasakis GN, Guggenheim B, Bostanci N. Down-regulation of NLRP3 inflammasome in gingival fibroblasts by subgingival biofilms: Involvement of Porphyromonas gingivalis. Innate Immun. 2013 Feb; 19 (1):3-9.

40. Grant RW, Dixit VD. Mechanisms of disease: inflammasome activation and the development of type 2 diabetes. Front Immunol. 2013; 4:50.

41. Allen EM, Matthews JB, DJ OH, Griffiths HR, Chapple IL. Oxidative and inflammatory status in type 2 diabetes patients with periodontitis. J Clin Periodontol. 2011; 38:894-901.

42. Deshpande K, Jain A, Sharma R, Prashar S, Jain R. Diabetes and periodontitis. J Indian Soc Periodontol. 2010 Oct; 14 (4):207-212.
43. Manouchehr-Pour M., Spagnuolo PJ., Rodman HM., Bissada NF. Comparison of neutrophil chemotactic response in diabetic patients with mild and severe periodontal disease. J Periodontol. 1981 Aug; 52 (8):410-415.

44. Sakallioglu EE, Ayas B, Lütfioglu M, Keles GC, Açikgöz G, Firatli $\mathrm{E}$. Gingival levels of monocyte chemoattractant protein-1 (MCP-1) in diabetes mellitus and periodontitis: an experimental study in rats. Clin Oral Investig. 2008 Mar; 12(1):83-9.

45. Gupta M, Chaturvedi R, Jain A. Role of monocyte chemoattractant protein-1 (MCP-1) as an immune-diagnostic biomarker in the păthogenesis of chronic periodontạl disease. Cytokine. 2013 Mar; 61 (3):892-7.

46. Salvi GE, Carollo-Bittel B, Lang NP. Effects of diabetes mellitus on periodontal and peri-implant conditions. Update on associations and risks. J Clin Periodontol. 2008; 35 (Suppl.8): 398-409.

47. Salvi GE, Beck JD, Offenbacher S. PGE2, IL-1 $\beta$ and TNF $\alpha$ responses in diabetics as modifiers of periodontal disease expression. Ann Periodontol. 1998 Jul; 3 (1):40-50.

48. ReesTD. Periodontal management of the patient with diabetes mellitus. Periodontology 2000, 2000; 23:63-72.

49. Haffajee AD, Socransky SS, Gunsolley JC Systemic anti-infective periodontal therapy. A systematic review. Ann Periodontol. 2003 Dec; 8 (1):115-181.

50. Guerrero A, Griffiths GS, Nibali L, Suvan J, Moles DR, Laurell $L$, Tonetti MS. Adjunctive benefits of systemic amoxicillin and metronidazole in non-surgical treatment of generalized aggressive periodontitis: a randomized placebo-controlled clinical trial. J Clin Periodontol. 2005 Oct; 32 (10):1096-1107.

51. Winkel EG, Van Winkelhoff AJ, Timmerman MF, Van der Velden $U$, Van der Weijden GA Amoxicillin plus metronidazole in the treatment of adult periodontitis patients. A double-blind placebo-controlled study. J Clin Periodontol. 2001 Apr; 28 (4):296-305.

52. Rifkin BR, Vernillo AT, Golub LM. Blocking periodontal disease progression by inhibiting tissue-destructive enzimes: a potential therapeutic role for tetracyclines and their chemically-modified analogs. J Periodontol. 1993 Aug; 64 (8 Suppl):819-827.

53. Grossi SG, Skrepcinski FB, De Caro T, Robertson DC, Ho AW, Dunford RG, et al.Treatment of periodontal disease in diabetics reduces glycated hemoglobin. J Periodontol. 1997; 68:713-9.

54. Grossi SG, Skrepcinski FB, DeCaroT, Zambon JJ, Cummins D, Genco RJ. Response to periodontal therapy in diabetics and smokers. J Periodontol. 1996 Oct; 67(10 Suppl):1094-1102.

55. Serrano C, Pérez C, Sabogal D Effect of periodontal therapy on metabolic control and an inflammatory mediator in type 2 diabetic subjects: a report on 17 consecutive cases. J Int Acad Periodontol. 2012 Apr; 14 (2):26-34

56. Tervonen T Knuuttila M, Pohjamo L, Nurkkala H. Immediate response to non-surgical periodontal treatment in subjects with diabetes mellitus. J Clin Periodontol. 1991 Jan; 18 (1): 65-68.

57. Westfelt E, Rylander H, Blohmé G, Jonasson P, Lindhe J. The effect of periodontal therapy in diabetics. Results after 5 years. J Clin Periodontol. 1996 Feb; 23 (2):92-100.

58. Karialainen KM, Knuuttila ML, Von Dickhoff KJ. Association of the severity of periodontal disease with organ complications in type 1 diabetic patients. J Periodontol. 1994 Nov; 65 (11):1067-1072.

59. Chen L, Wei B, Li J, Liu F, Xuan D, Xie B, Zhang J. Association of periodontal parameters with metabolic level and systemic inflammatory markers in patients with type 2 diabetes. J Periodontol. 2010 March; 81 (3):364-371.

60. Saito T, Shimazaki Y, Kiyohara Y, Kato I, Kubo M, lida M, KogaT. The severity of periodontal disease is associated with the development of glucose intolerance in non-diabetics: the Hisayama study. J Dent Res. 2004 Jun; 83 (6):485-490.

61. Donahue RP, Wu T. Insulin resistance and periodontal disease: an epidemiologic overview of research needs and future directions. Ann Periodontol 2001; 6: 119-124. 